\title{
Malignant melanoma involving the small bowel
}

\author{
B.G. Wilson and J.R. Anderson
}

Department of Surgery, Royal Victoria Hospital, Belfast BT12 6BA, Northern Ireland, UK.

\begin{abstract}
Summary: Small bowel is the commonest site of gastrointestinal metastases from cutaneous malignant melanoma. Five patients with malignant melanoma involving the small bowel are reported. One patient was operated on for suspected acute appendicitis, two patients for gastrointestinal bleeding and two patients for small bowel obstruction. Two patients remain well 4 and 5 years after surgery.
\end{abstract}

\section{Introduction}

Cutaneous malignant melanoma is an unpredictable tumour and may metastasize to almost any organ. The recorded post-mortem incidence of metastatic involvement of the gastrointestinal tract is $58 \%$ for small bowel, $53 \%$ for pancreas, $26 \%$ for stomach and $15 \%$ for gallbladder (Das Gupta \& Brasfield, 1964). It is, however, uncommon for gastrointestinal metastases to present during the clinical course of the disease. The clinical records of all patients diagnosed at the Pathology Departments of the Belfast City and Royal Victoria Hospitals and Altnagelvin Hospital, Londonderry, as having malignant melanoma involving small bowel since 1970 were reviewed. The presentation, results of surgery and outcome are discussed.

\section{Case reports}

Case 1

A 42 year old woman presented with signs and symptoms suggestive of appendicitis. Five years previously a malignant melanoma of the left lower leg had been removed and 5 months later she underwent a block dissection of the inguinal region where two lymph nodes were found to be infiltrated.

At laparotomy, two polypoidal tumours were found in the terminal ileum $9 \mathrm{~cm}$ apart, measuring 2 and $3 \mathrm{~cm}$ in diameter respectively. The appendix was normal. There was no other evidence of abdominal metastases, and in particular the liver was normal to palpation. The ileum was resected, and histopathological examination revealed that both tumours were amelanotic melanomas. The lymph nodes in the specimen

Correspondence: B.G. Wilson, F.R.C.S., Department of Surgery, The Queen's University of Belfast, Institute of Clinical Science, Grosvenor Road, Belfast BT12 6BJ

Accepted: 1 November 1985 were not involved with tumour. The patient made an uneventful post-operative recovery and remains well 5 years following surgery.

\section{Case 2}

A 54 year old man presented with weight loss and melaena. On examination, a mass in the left upper quadrant was easily palpable. Upper gastrointestinal endoscopy and barium enema were normal. Seventeen years earlier the patient had a malignant melanoma excised from the left side of the chest and 10 years following that procedure had a local recurrence excised from the same area.

At laparotomy a $14 \mathrm{~cm}$ diameter melanotic tumour of the mid-jejunum was found adherent to the renal capsule, splenic flexure, omentum and with obvious lymph node involvement. The patient underwent an en bloc dissection of jejunum, omentum and left colon with restoration of gastrointestinal continuity at the time of laparotomy. His immediate post-operative recovery was uneventful but he died 4 months later from disseminated disease within the abdomen confirmed at post-mortem.

\section{Case 3}

A 58 year old man presented with melaena. Upper gastrointestinal endoscopy revealed a polyp in the second part of the duodenum with no stigmata of recent haemorrhage. Biopsy revealed an amelanotic melanoma. Chest X-ray showed a mass in the left hilum, and sputum cytology revealed cells consistent with a diagnosis of an amelanotic melanoma. Laparotomy was carried out because of continuing haemorrhage. There were 36 polypoidal lesions found throughout the jejunum, some of which showed central umbilication and almost certainly these were the cause of the patient's melaena. The duodenal polyp

(C) The Fellowship of Postgraduate Medicine, 1986 
was excised together with all the jejunum containing the multiple polyps. Histopathological examination of the resected specimen showed that the multiple polyps within the jejunum were melanotic melanomas, unlike the duodenal tumour which was amelanotic. The immediate post-operative course was uneventful but the patient died 3 months later from progression of disease.

There was no history of a previously excised skin lesion, and no clinical evidence on careful examination of a cutaneous melanoma.

\section{Case 4}

A 55 year old man presented with simple small bowel obstruction. Eighteen months before admission a malignant melanoma had been excised from the left occipital region of the scalp.

At laparotomy two tumours were found. The obstructing lesion was an infiltrative annular tumour in the ileum measuring $8 \mathrm{~cm}$ in diameter. A second, more proximal polypoidal tumour measuring $6 \mathrm{~cm}$ in diameter, was found projecting into the lumen. Both tumours were resected and the immediate postoperative period was uneventful. Histopathological examination of the resected specimens showed the typical features of a melanotic melanoma with tumour deposits in the draining lymph nodes. The patient remained well for 3 days and then suddenly had a cardio-pulmonary collapse and died. Post-mortem examination was not carried out.

\section{Case 5}

A 60 year old woman presented with a small bowel obstruction. At laparotomy a $6 \times 4 \mathrm{~cm}$ infiltrative dark black tumour was found in the ileum which was resected. Histopathological examination showed the typical features of a melanotic melanoma, but the local lymph nodes were not involved. This patient remains well 4 years following surgery. There is no history of skin lesion having been excised, and no evidence of any orbital or other malignant melanoma.

\section{Discussion}

The incidence of gastrointestinal metastatic melanoma presenting in living patients is low. Das Gupta \&
Brasfield (1964) have reported only nine cases in their series of one thousand melanoma patients. Post-mortem studies, however, demonstrate a much higher incidence of intestinal involvement and the explanation for this difference is unknown. Patients with intestinal melanomas usually present with obstruction or gastrointestinal bleeding, as occurred in four of the cases in the present study. Weight loss is usually a prominent feature, but was only noted in one of our patients (case 2).

Macroscopically, two distinct types of lesion are recognized. Polypoidal tumours are frequently multiple and often amelanotic whilst infiltrative lesions are usually single and melanotic, the former being more common. Willbanks \& Folegman (1970) have shown that patients with infiltrative lesions have a better prognosis than those with multiple polypoidal melanomas, although the reasons for this are obscure. In the present study two of the five patients are alive, four and five years following surgery, after resection of one infiltrative lesion and two polypoidal lesions respectively. Polypoidal and infiltrative lesions may co-exist in the same patient (case 4); these patients probably have a prognosis similar to the polypoidal tumour group.

Two patients in the present study had no previous history of excision of a cutaneous melanoma or any clinical evidence at the time of presentation of such a lesion. This has been previously noted and has led too speculation that intestinal melanoma may on occasion be primary (Tabaie et al., 1984). It is well recognized that primary cutaneous tumours may spontaneously regress, sometimes in the presence of regional metastases (Smith \& Stehlin, 1965). Melanoblasts are not found in small or large intestine and it is therefore unlikely that melanomas of the intestine are primary (Willis, 1948).

Metastatic intestinal melanoma should be considered in any patient presenting with gastrointestinal symptoms, and a previous history of a melanotic skin lesion. When found, adequate local excision will occasionally be rewarded with long term survival.

\section{Acknowledgements}

The authors would like to thank Professor T.G. Parks for comments on the manuscript and also the consultant surgeons who gave permission to report these cases.

\section{References}

DAS GUPTA, T.K. \& BRASFIELD, R.D. (1964). Metastatic melanoma of the gastrointestinal tract. Archives of Surgery, 88, 969.

SMITH, J.L. JR. \& STEHLIN, J.S. JR. (1965). Spontaneous regression of primary malignant melanomas with regional metastases. Cancer, 18, 1399. 
TABAIE, H.A., CITTA, R.J., GELLO, L., BIONDI, R.J., MEOLI, F.G. \& SILVERMAN, D. (1984). Primary malignant melanoma of the small intestine. Report of a case and discussion of the APUD cell concept. Journal of the American Osteopathic Association, 83, 374.

WILLBANKS, O.L. \& FOLEGMAN, M.J. (1970). Gastrointes- tinal melanosarcoma. American Journal of Surgery, 120, 602.

WILLIS, R.A. (1948). In Pathology of Tumours, p. 915. Butterworth \& Co. Ltd.: London and C.V. Mosby Co.: St. Louis. 